\title{
Ocean energy harvester based on piezoelectric VIV using different oscillators
}

\author{
Min Liu ${ }^{1,2}$, Hui Xia ${ }^{1,2^{*}}$, Guoqiang Liu ${ }^{1,2^{*}}$, Dong Xia ${ }^{1,2}$ \\ ${ }^{1}$ School of Electronic Electrical and Communication Engineering, University of Chinese Academy of Sciences, Beijing, 100190, China \\ ${ }^{2}$ Institute of Electrical Engineering, Chinese Academy of Sciences, Beijing, 100190, China
}

\begin{abstract}
A finite element fluid-solid coupling model for ocean energy harvester based on piezoelectric vortex-induced vibration(VIV) is established. Given that the Karman Vortex Street is generated after the fluid passes through the vibrator. The model includes the conversion of water flow energy to VIV energy and the capture of electrical energy by piezoelectric devices. And the output voltage curve is obtained by coupling with piezoelectric beam. Based on the fluid-solid coupling calculation, the dynamic response characteristics of the oscillator under different parameters such as shape of oscillators and fluid velocity are studied. The voltage output of piezoelectric beam in cylindrical, semi-cylindrical and regular triangular oscillators is analyzed. Simulation results show that the output voltage and pressure difference are largest in regular triangular oscillator system compared with the cylindrical and semi-cylindrical system. When changing fluid velocity, it is found that the higher the velocity of the water fluid be, the higher the output voltage be. When the given fluid velocity reaches $1 \mathrm{~m} / \mathrm{s}$, the maximum output voltage of cylindrical, semi-cylindrical and regular triangular piezoelectric energy harvesters reaches $0.045 \mathrm{~V}, 0.08 \mathrm{~V}$, and $0.085 \mathrm{~V}$ respectively. Under the same fluid velocity, change the ratio of height and width of oscillator, and find that the higher ratio of height and width of oscillator is more suitable to harvest the energy of VIV.
\end{abstract}

\section{Introduction}

The efficiency of renewable energies has grown significantly in recent years and ocean energy has been one of the most important responsibilities. The mechanical energy in natural environment can be converted into electricity supplied for small equipment due to the micro-energy technology.

The vibrations induced in a body by vortices are known as the vortex-induced vibration(VIV) phenomena[1]. The VIV effect is used to convert the mechanical energy of fluid into vibration energy. Allen and Smith et al.[2] designed the eel energy collector, which converted the flow energy into the vibration mechanical energy. Sarpakay et al.[3] derived the principle of VIV, did the numerical analysis and the experimental researches. Gabbai and Benaroya et al.[4] carried out the numerical researches and experimental verifications based on cylindrical vibrator. The team from the University of Michigan[1,5] analyzed the dynamic response of VIV at different Reynolds numbers and designed a water flow energy harvesting device. Piezoelectric[6] and electromagnetic[7] capture devices are the two most effective ways to convert vibration energy into electrical energy. In recent years, the technology of using piezoelectric effect to collect the surrounding environment energy has been widely concerned with the development of piezoelectric materials.
For example, Meiling et al.[8,9] realized dimension reduction numerical analysis for the generation efficiency of piezoelectric energy capture device. Abdelkefi et al.[10] showed that the energy conversion efficiency of piezoelectric beam was related to the amplitude of cantilever beam. Xiaobiao SHAN and his team[11] built a piezoelectric and electromagnetic energy harvesting model, which broadened the frequency band of the vibration and improves the power generation efficiency.

The dynamic response of VIV is closely related to the shape of the oscillator and the fluid velocity. In order to optimize the energy harvester of VIV, this paper takes the combination of half cylinder vibrator and $\mathrm{ZnO}$ piezoelectric material as an example to study the output voltage and power when the shape of the oscillator and the flow velocity of the fluid change.

\section{Modeling of piezoelectric energy harvester}

A piezoelectric energy harvesting model is established combined with the relevant literature [12]. The vortex shedding happens with forces perpendicular to the incident water flow direction with periodicity. The forces cause the vibration of piezoelectric beam and the vibration energy is converted into electrical energy through piezoelectric effect. As shown in Figure 1, the piezoelectric beam is located behind the center of the

*Email: xiahui@mail.iee.ac.cn, liuguoqiag@mail.iee.ac.cn 
oscillator. The beam is composed of three layers. The upper and lower layers are substrate layers, and the middle layer is piezoelectric layer. The piezoelectric material is deformed by the vibration force and outputs the voltage signal. The piezoelectric beam and the external resistance form a closed circuit. The electrical energy generated by the piezoelectric beam supplies power for the external circuit or is stored.

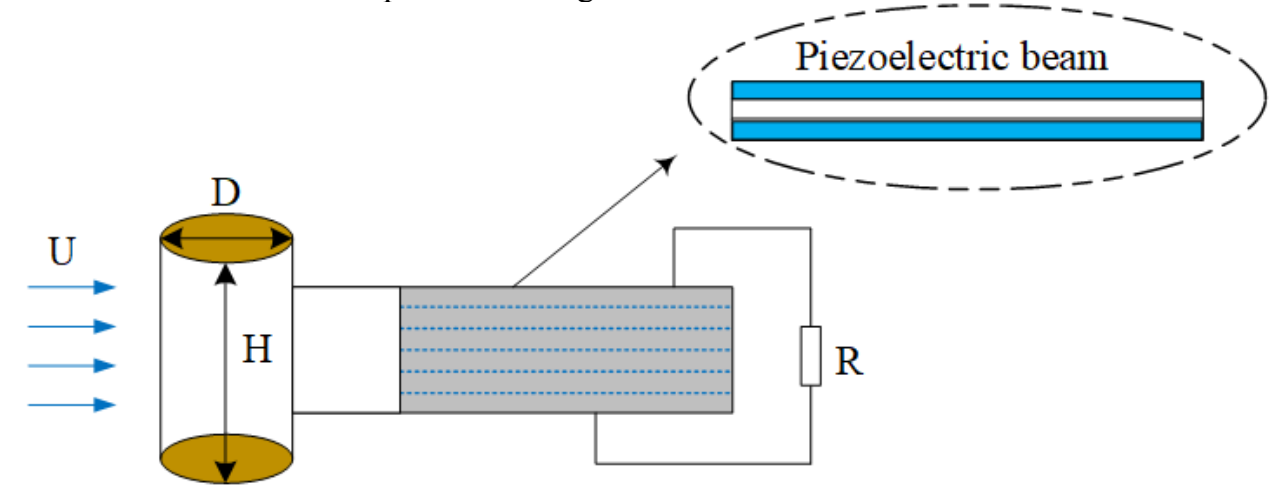

Figure 1. Modeling of piezoelectric energy harvester

The differential equation of motion for vibration is as equation (1), where $\ddot{y}, \dot{y}, y$ are the acceleration, velocity and displacement of the vibration system respectively. $m_{\text {osc }}$ is the vibration mass, including the mass of cylindrical body and the piezoelectric beam. $C$ is the mechanical damping coefficient. $K$ is the stiffness.

$m_{o s c} \ddot{y}+C \dot{y}+K y=F(t)$

The main forces of vibrator in water includes inertia force and viscous force, which can also be expressed by drag force $F_{D}$ and lift force $F_{L}$, where $C_{D}$ and $C_{L}$ are dimensionless drag coefficient and lift coefficient respectively. $A_{0}$ is projected area.

$$
F_{D}=\frac{\rho v^{2} C_{D} A_{0}}{2}, F_{L}=\frac{\rho v^{2} C_{L} A_{0}}{2}
$$

The vibration excitation of the vibrator can be equivalent to a spring-mass-damper system, as shown in Figure 2. It shows the diagram where a mass $m_{\text {osc }}$ oscillates under the action of the fluid velocity $U$ and it is connected to a spring of constant $K$ and a damper of constant $C$. The damper $C$ includes mechanical damping and piezoelectric damping.

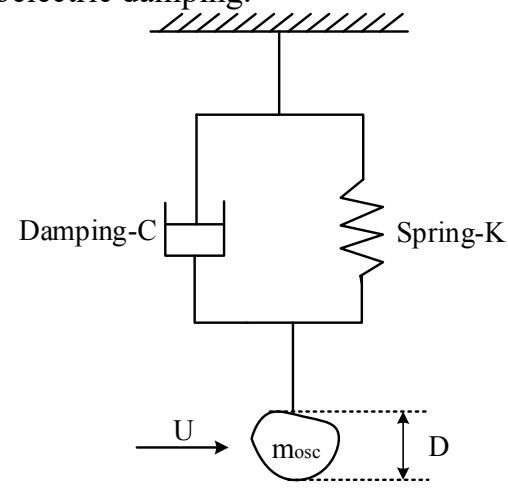

Figure 2. Spring-Mass-Damper diagram

According to the motion differential equation of VIV, the vibration equation of equivalent spring mass damper system can be obtained as equation (3), where $\omega_{0}$ is angular frequency of natural vibration without damping and $\zeta$ is total damping coefficient of the system. $\omega_{0}$ and $\zeta$ can be expressed as equation (4).

$$
\begin{aligned}
& \ddot{y}(t)+2 \zeta \omega_{0} \dot{y}(t)+\omega_{0}^{2} y(t)=A \omega^{2} \cos (\omega t) \\
& \omega_{0}=\sqrt{\frac{K}{m}}, \zeta=\frac{C}{2 m_{o s c} \omega_{0}}
\end{aligned}
$$

The solution of the vibration equation of can be expressed as equation (5).

$$
y(t)=\frac{A\left(\omega / \omega_{0}\right)^{2}}{\sqrt{\left(1-\left(\omega / \omega_{0}\right)^{2}\right)^{2}+\left(2 \zeta\left(\omega / \omega_{0}\right)\right)^{2}}} \cos (\omega t)
$$

There is a constant of proportionality $S t$ between the average velocity of the water flow $U$, the inverse of its characteristic length $\mathrm{D}$ and the frequency of vortex shedding $f$ :

$$
f=S_{t} U / D
$$

$\omega$ can be found out as based on $f, \omega=2 \pi f$. According to the beam theory, the strain in the piezoelectric layer is as equation (7), where $u_{b}$ is the amplitude of piezoelectric beam.

$S(x, t)=-y_{b} u_{\mathrm{b}}(x, t)$

The charge $Q$ on the electrode can be obtained by integrating the potential shift vector $D$ in the piezoelectric layer. $D$ and $Q$ can be expressed by equation (8) and equation (9), where $d_{31}$ is the piezoelectric constant when piezoelectric materials work in the mode of $d_{31}$. $E_{b}$ is the equivalent Young's modulus of the piezoelectric cantilever. $w$ and $l_{11}$ are the thickness and length of the piezoelectric beam respectively.

$D=d_{31} E_{b} S$

$Q=w \int_{0}^{l_{11}} D d x$ 
Finally, the output voltage of piezoelectric beam can be expressed as equation (10), where $C_{b}$ is the capacitance formed by the upper and lower electrodes of the piezoelectric material, and $C_{b}=\varepsilon l \omega / h p . \varepsilon$ is the dielectric constant.:

$V=\frac{Q}{C_{b}}$

\section{Finite element analysis and results}

\subsection{Response of VIV}

Vortex shedding can be produced when Reynolds number is more than 40. As shown in Figure 3, semi-cylindrical, cylindrical and regular triangle blunt bodies are selected to be the oscillators.

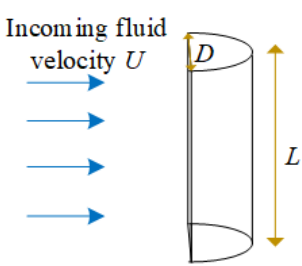

(a)

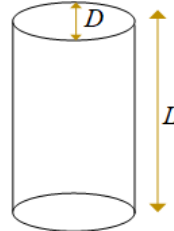

(b)

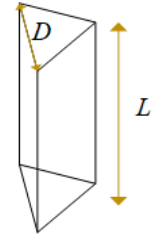

(c)
Figure 3. Different oscillators: (a) semi-cylindrical oscillator; (b) cylindrical oscillator; (c) regular triangle oscillator.

$\mathrm{ZnO}$ is selected as the piezoelectric material. The oscillators are made of light wood. The characteristics of fluid and the parameters of piezoelectric beam are listed in Table 1.

Table 1. property parameters of the system

\begin{tabular}{cc}
\hline Parameters & Values \\
\hline Dynamic viscosity of water flow, $\mu(\mathrm{Pa} \cdot \mathrm{s})$ & $1.6128 \times 10^{-3}$ \\
Density of water flow, $\rho\left(\mathrm{kg} / \mathrm{m}^{3}\right)$ & 1030 \\
Characteristic length, $D(\mathrm{~m})$ & $0.01,0.02$ \\
Thickness of piezoelectric beam, $w(\mathrm{~m})$ & 0.001 \\
Length of piezoelectric beam, $l_{11}(\mathrm{~m})$ & 0.03 \\
Dielectric constant of piezoelectric beam, $\varepsilon$ & $\{8.5446,8.5446,10.204\}$ \\
\hline
\end{tabular}

According to the parameters in the Table 1, a VIV model composed of fluid and vibrator is built to realize the fluid-solid coupling. The response of VIV is closely related to the size of oscillator, the shape of oscillator and the incoming fluid velocity, which affect the Reynolds number, the frequency of vortex shedding, and pressure

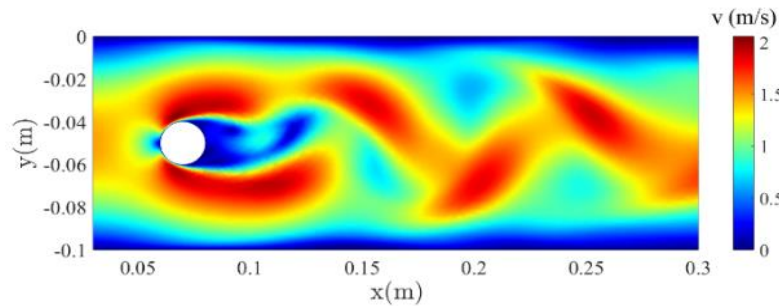

(a)

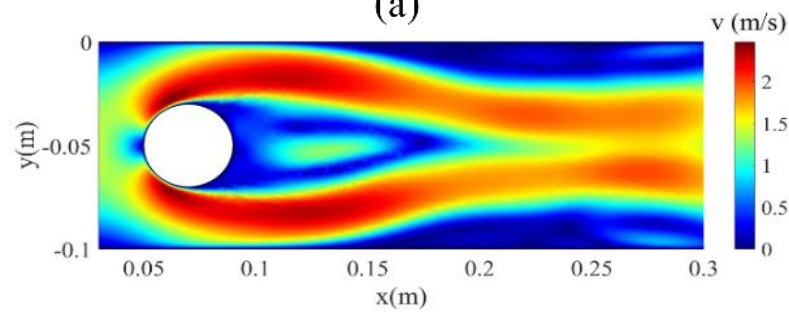

(c) distribution. Taking the cylindrical oscillator as an example, we analyze the influence of diameter of oscillator on the velocity of vortex shedding when the diameter $D$ is $0.01 \mathrm{~m}$ and $0.02 \mathrm{~m}$ respectively and the incoming fluid velocity $U$ is $1 \mathrm{~m} / \mathrm{s}$.

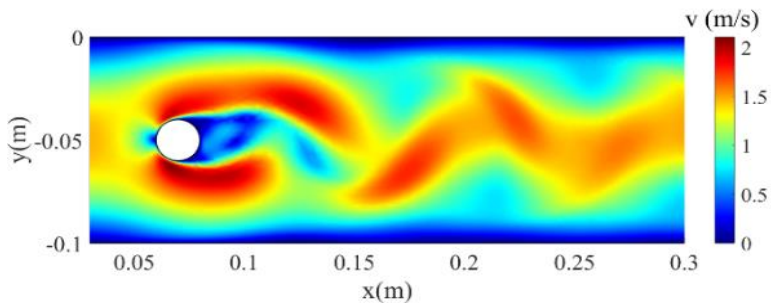

(b)

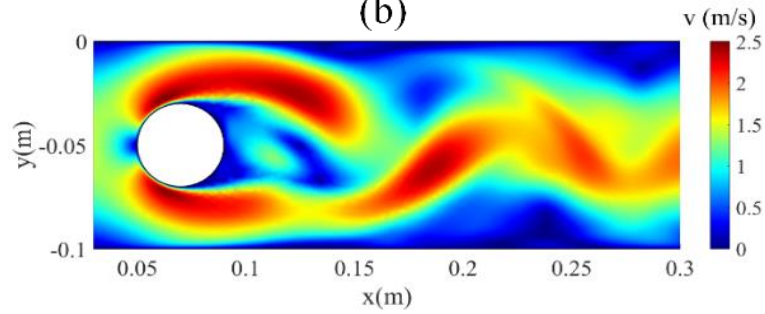

(d)

Figure 4. Velocity distribution of cylindrical vibrator with different diameters at different times: (a) $D=0.01 \mathrm{~m}, \mathrm{t}=0.6 \mathrm{~s}$; (b) $\mathrm{D}=0.01 \mathrm{~m}$, $\mathrm{t}=5 \mathrm{~s}$; (c) $\mathrm{D}=0.02 \mathrm{~m}, \mathrm{t}=0.6 \mathrm{~s}$; (d) $\mathrm{D}=0.02 \mathrm{~m}, \mathrm{t}=5 \mathrm{~s}$.

It can be seen from Figure 4, the smaller characteristic diameter of the oscillator, the shorter the time to form stable vortex shedding after the vibration. When the diameter of the cylindrical vibrator is twice larger than the original one, the velocity of vortex increases at the same incoming fluid velocity and at the same time. We select three typical structures of triangle, semicircle and cylindrical with the same characteristic length $D$ for simulation, and get the pressure distribution diagrams as shown in Figure 4. 

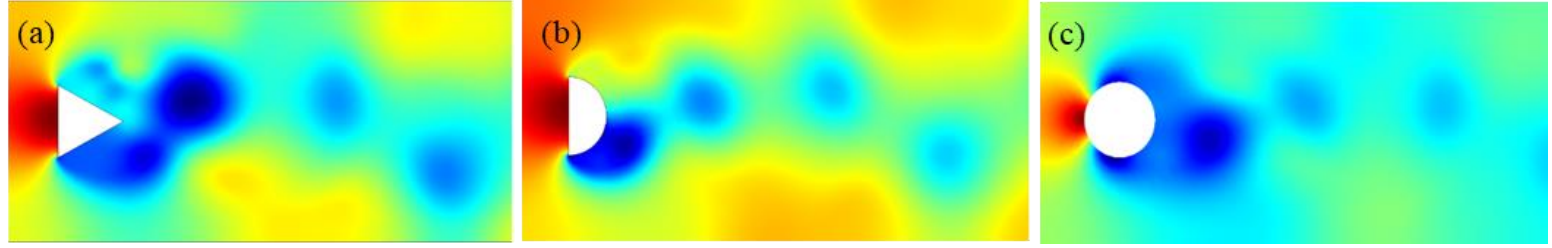

Figure 5. Pressure distribution diagram of different vibrators, $U=0.61 \mathrm{~m} / \mathrm{s}, \quad t=6 \mathrm{~s}:$ (a)triangle oscillator; (b) semi-cylindrical oscillator; (c) cylindrical oscillator.

From Figure 5, the pressure distributions of triangle and semicircle vibrators are similar and they are larger than the pressure distribution of the cylindrical vibrator. Considering that the circular structure can adapt to the flow in all directions, most scholars use the cylindrical vibrator in the deep sea environment. The piezoelectric energy harvesting system assumes that the piezoelectric beam is fixed directly behind the center of vibrator, so it assumes that the flow direction is only one direction and different oscillators can be used.

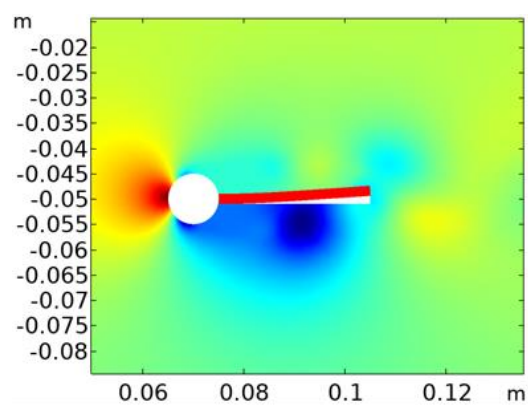

(a)

\subsection{Output of the system}

The output voltage of the energy harvester is proportional to the deformation of piezoelectric beam. The larger the pressure difference between the two sides of the piezoelectric beam be, the greater its deformation be. Cylindrical and semi-cylindrical oscillators are used for comparison, and Figure 6 shows the pressure distribution of vortex street formed under the two structures. From Figure 7 , the output voltage of semi-cylindrical, regular triangular and cylindrical energy harvester is gained and compared.

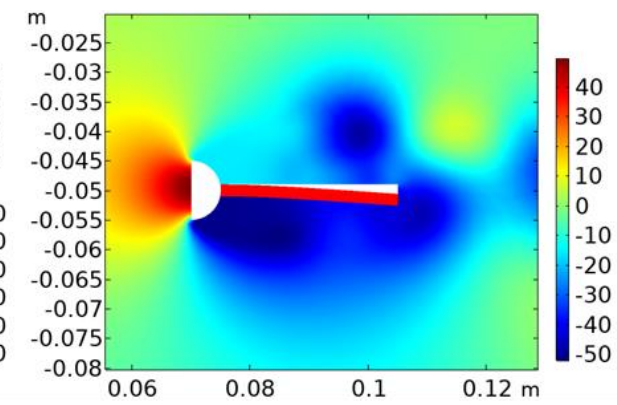

(b)

Figure 6. Pressure distribution diagram of different vibrators with piezoelectric beam: (a) cylindrical oscillator; (b) semi-cylindrical oscillator.
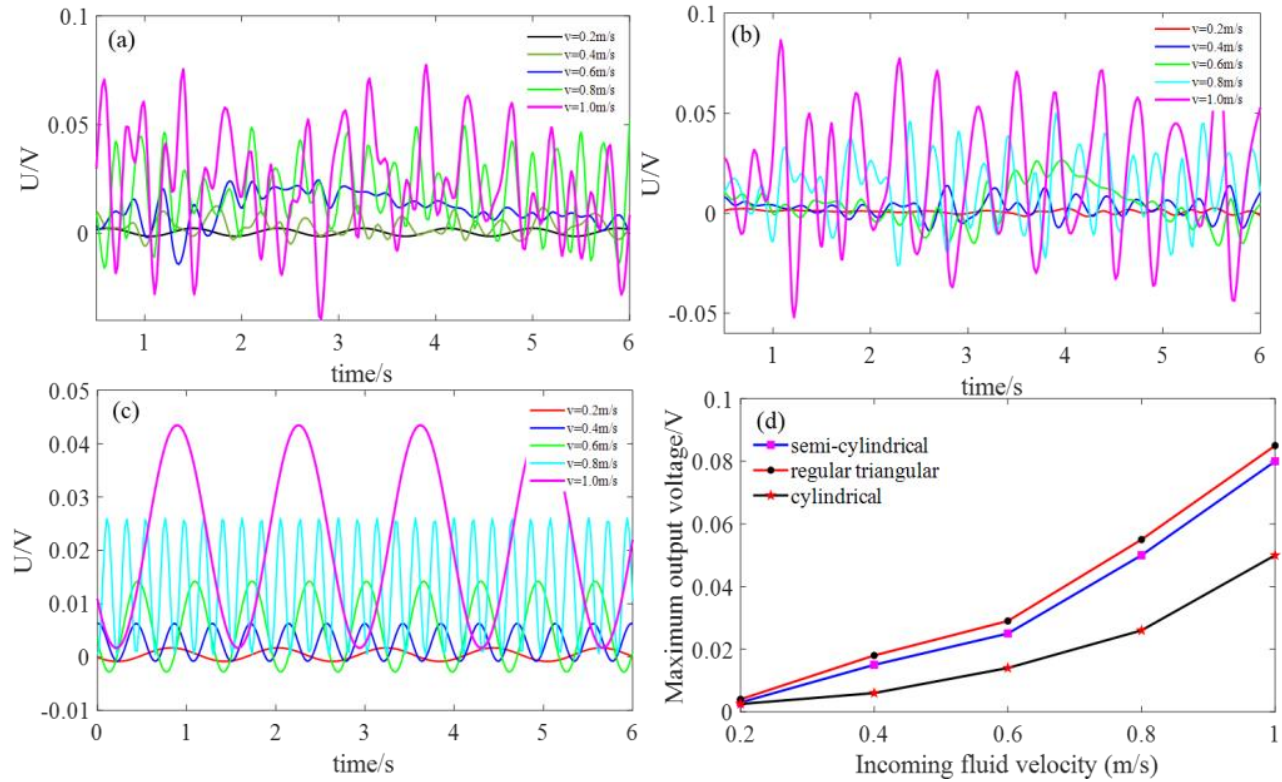

Figure 7. Relationship between the output voltage and time for different fluid velocities with different shape of vibrators: (a) semicylindrical oscillator; (b) regular triangular oscillator; (c) cylindrical oscillator; (d) relationship between the maximum output voltage of three structures and the fluid velocity. 
As shown in Figure 6, the piezoelectric beam is fixed behind the center of the oscillator with a given incoming fluid velocity of $0.2 \mathrm{~m} / \mathrm{s}$. The characteristic length of cylinder and semi-cylinder in the system is $0.01 \mathrm{~m}$. By comparing the pressure distribution at the same time of two kinds of vibrator, the deformation of the piezoelectric beam and the overall pressure of semi-cylindrical vibrator is larger than those of cylindrical vibrator. Figure 7 illustrates the relationship between the transient output voltage and time for different fluid velocities with semicylindrical oscillator (Figure 7a), regular triangular oscillator (Figure 7b), cylindrical oscillator (Figure 7c). The fluid velocity is selected $0.2,0.4,0.6,0.8$ and $1 \mathrm{~m} / \mathrm{s}$ because the energy harvesting system is used in deep sea. The relationships show that the maximum output voltage appears on the curve of regular triangular oscillator, which reaches $0.085 \mathrm{~V}$ at the fluid velocity of $1 \mathrm{~m} / \mathrm{s}$. The output voltage of semi-cylindrical oscillator and cylindrical oscillator is $0.08 \mathrm{~V}, 0.045 \mathrm{~V}$ respectively. The stability of the output voltage is better when the cylindrical oscillator is adopted.

\section{Conclusions}

By means of COMSOL Multiphysics, a two-dimensional piezoelectric energy harvesting system with VIV is numerically simulated. The influence of the characteristic length and structure of the cylindrical vibrator are emphatically studied on the velocity and pressure distribution of the VIV dynamic response. Furthermore, the influence of the oscillator shape and the fluid velocity on the pressure distribution and the output voltage of the energy harvesting system is studied.

It is found that when the characteristic length of oscillator is smaller, the time for the fluid to form stable vortex shedding is shorter. When the characteristic length of the cylindrical oscillator increases, the velocity of the whole flow field increases at the same fluid velocity and at the same time. The pressure and output voltage produced by the semi-cylindrical oscillator are larger by comparing the cylindrical and semi-cylindrical oscillator. We compared the influence of fluid velocity on output voltage with semi-cylindrical, regular triangular and cylindrical oscillator. The output voltage increases with the increase of fluid velocity. As a whole, when the fluid velocity reaches $1 \mathrm{~m} / \mathrm{s}$, the maximum output voltage can reach $0.085 \mathrm{~V}$ using regular triangular oscillator.

\section{References}

1. Lee J.H., Xiros N., Bernitsas M M . (2011) Virtual damper-spring system for VIV experiments and hydrokinetic energy conversion. Ocean Engineering, 38(5-6):732-747.

2. Allen J.J, Smits A.J. (2001) ENERGY HARVESTING EEL. Journal of Fluids \& Structures, 15(3-4):629-640.

3. Sarpkaya T. (2004) A critical review of the intrinsic nature of vortex-induced vibrations. Journal of Fluids \& Structures, 19(4):389-447.Thompson, J.N. (1984)
Insect Diversity and the Trophic Structure of Communities. In: Ecological Entomology. New York. pp. 165-178.

4. Gabbai R.D , Benaroya H. (2005) An overview of modeling and experiments of vortex-induced vibration of circular cylinders. Journal of Sound and Vibration, 282(3-5):575-616. .

5. Lee J.H, Bernitsas M.M. (2011) High-damping, highReynolds VIV tests for energy harnessing using the VIVACE converter. Ocean Engineering, 38(16):1697-1712.

6. Priya S. (2007) Advances in energy harvesting using low profile piezoelectric transducers. Journal of Electroceramics, 19(1):167-184.

7. Wang D.A , Chang K H . (2010) Electromagnetic energy harvesting from flow induced vibration. Microelectronics Journal, 41(6):356-364.

8. Zhu M., Leighton G. (2008) Dimensional reduction study of piezoelectric ceramics constitutive equations from 3-D to 2-D and 1-D. IEEE Transactions on Ultrasonics Ferroelectrics \& Frequency Control, 55(11):2377-83.

9. Zhu M., Leighton G. (2008) Dimensional reduction study of piezoelectric ceramics constitutive equations from 3-D to 2-D and 1-D. IEEE Transactions on Ultrasonics Ferroelectrics \& Frequency Control, 55(11):2377-83.

10. Abdelkefi A., Nayfeh A.H., Hajj M.R.. (2011) Modeling and analysis of piezoaeroelastic energy harvesters. Nonlinear Dynamics, 67(67):925-939.

11. Shan X.B., Guan S.W., Liu Z.S. (2013) A new energy harvester using a piezoelectric and suspension electromagnetic mechanism. Journal of Zhejiang University-Science A(Applied Physics \& Engineering), 14(12):890-897.

12. Naseer R., Dai H.L., Abdelkefi A. (2017) Piezomagnetoelastic energy harvesting from vortexinduced vibrations using monostable characteristics. Applied Energy, 203:142-153. 